\title{
Chondroblastic osteosarcoma arising in the maxilla mimicking the radiographic and histological characteristics of cemento-osseous lesions: A case report
}

\author{
BIN-BIN LI* , JIAN-YUN ZHANG* ${ }^{*}$ and YAN GAO \\ Department of Oral Pathology, Peking University School and Hospital of Stomatology, Beijing 100081, P.R. China
}

Received July 14, 2016; Accepted February 10, 2017

DOI: $10.3892 / \mathrm{mco} .2017 .1219$

\begin{abstract}
Osteosarcomas of the jaw are comparatively rare and represent only $2-10 \%$ of all osteosarcomas. We herein present a rare case of an osteosarcoma exhibiting the radiographic and histological characteristics of cemento-osseous lesions in the alveolar ridge of the maxilla. A 53-year-old male patient presented with the complaint of gradual swelling of the left maxilla over 4 years. Radiography revealed an ill-defined radioopaque mass, intimately associated with the apices of the involved teeth, without a periosteal reaction. Microscopically, a cementicle-like structure was identified in the alveolar bone. In addition, the lesion exhibited typical characteristics of chondroblastic osteosarcoma in the body of the maxilla. The tumor contained abundant osteoid and cartilage intimately associated with anaplastic tumor cells. The cartilage displayed malignant-appearing cells in lacunae, and there was crowding at the periphery of the lobule where the spindle cells formed sheets. The differential diagnosis included primary osteosarcoma, concurrent cemento-osseous dysplasia and osteosarcoma, or a secondary osteosarcoma based on a pre-existing cemento-osseous lesion. The presence of the cementicle-like structure in the alveolar bone and the involvement of the periodontal ligament and alveolar bone proper were unique in our case. The general invasive growth pattern and the abundance of the irregular tumor bone helped establish the diagnosis of primary osteosarcoma. This case may represent evidence of the pathogenesis of primary osteosarcoma in the jaw.
\end{abstract}

Correspondence to: Dr Yan Gao, Department of Oral Pathology, Peking University School and Hospital of Stomatology, 22 Zhongguancun South Avenue, Haidian, Beijing 100081, P.R. China

E-mail: gaoyan0988@163.com

*Contributed equally

Key words: osteosarcoma, jaw, cementum

\section{Introduction}

Osteosarcoma is a type of malignant bone tumor in which the neoplastic cells produce osteoid or bone (1). Osteosarcomas of the jaw are rare and represent only $2-10 \%$ of all osteosarcomas. Osteosarcoma of the head and neck region is the most common primary malignant bone tumor, representing $23 \%$ of all head and neck malignancies (2). Osteosarcoma arises more frequently in the maxilla compared with the mandible (3). Unlike the hallmark clinical presentation of pain in lesions of the long bones, the most characteristic symptom of jaw osteosarcoma is swelling. Similar with osteosarcoma of long bones, the dominant histological variant of osteosarcoma is the osteoblastic type, followed by the chondroblastic and fibroblastic types (4). However, the pathologist may encounter a potential diagnostic pitfall when an osteosarcoma of the jaw resembles a cemento-osseous lesion. We herein present a case of maxillary osteosarcoma mimicking the histological and radiographic characteristics of cemento-osseous lesions.

\section{Case report}

A 53-year-old male patient presented in May, 2014 with gradual swelling of the left side of the face over a period of 4 years. The patient's medical history was non-contributory. The physical examination revealed an uncircumscribed swelling of the left maxilla, with normal color of the overlying skin. A panoramic radiograph revealed an ill-defined radiopaque mass at the apices of the roots of the left maxillary bone, from the incisor to the second bicuspid (Fig. 1). The mass was unilateral and closely associated with the teeth roots; however, there was no root resorption in the involved teeth. Further computed tomography examination revealed that the size of the mass was $2.3 \times 2 \times 1.7 \mathrm{~cm}$. No periosteal reaction was observed, and no soft tissue extension component beyond the area of the cancellous and/or cortical bone destruction was identified radiographically. The lesion involved the left wall of the incisive canal and the left hard palate, resulting in a discontinuous labial and palatal alveolar bone plate (Fig. 2A). The corresponding nasal bottom and left wall of the maxillary sinus were eroded (Fig. 2B). Given its association with the teeth root and the diffuse borders, the most likely clinical diagnosis was a malignant tumor originating from cementum. The tumor was treated by radical resection of the left maxillary bone. 
The specimens were fixed in $10 \%$ buffered formalin, dehydrated through graded concentrations of ethanol and embedded in paraffin wax. The paraffin block was then cut in 4-mm sections that were stained with haematoxylin and eosin (H\&E).

Grossly, the tumor involved alveolar bone as well as bone from the body of the maxilla, from the first incisor to the proximal side of the first molar (Fig. 3). All the roots of the involved teeth were partially embedded in the tumor. The tumor appeared as pale, without clear borders from the surrounding bone tissue. The sectional area of the mass was $3.4 \times 3 \mathrm{~cm}$. Microscopically, the mass displayed a different appearance in the alveolar bone and in the body of the jaw. In the alveolar bone part, the tumor infiltrated the periodontal ligament and alveolar bone proper (Fig. 4A). Extensive cementicle-like structure and deposits of hypercellular cartilage were observed in the alveolar bone (Fig. 4B). However, this cementum-like material was intimately associated with the pleomorphic and hyperchromatic spindle cell component, and it infiltrated the trabeculae of the medullary bone (Fig. 4C), suggesting a malignant tumor. Interestingly, a continuous transition from the trabecular alveolar bone to the tumor was observed at the bony side of the periodontal ligament, strongly supporting the hypothesis that the tumor originated from bone, not from the periodontal ligament, and suggesting that the tumor would differentiate towards osteoid and not towards cementum-like material.

In the bone of the maxilla, the mass exhibited the morphological characteristics of a typical chondroblastic osteosarcoma. The tumor contained a relatively equal distribution of abnormal osteoid and chondroid, intimately associated with anaplastic tumor cells (Fig. 5A). Islands of osteoid were observed within the cartilage. The tumor bone exhibited a woven or basket weave pattern and was strongly hematoxylinophilic, unlike the uniform lamellar pattern of normal bone. The cartilage displayed malignant-appearing cells in lacunae, and there was crowding at the periphery of the lobule, where spindle cells were arranged in sheets (Fig. 5B).

\section{Discussion}

Osteosarcoma is characterized by the osteoid production by tumor cells. Osteosarcoma of the jaw is associated with certain specific characteristics, such as a higher prevalence in the maxilla and male predominance. Wang et al reported that all maxillary de novo osteosarcomas arose from the alveolar ridge (5). The mass in the present case involved both the alveolar ridge and the body of the maxilla, possibly due to long-term growth leading to extension of the mass from the alveolar ridge to the body.

The differential diagnosis between lesions with hard tissue formation may be problematic in the oral area. These lesions are associated with the formation of bone, cartilage or cementum. Among these, osteosarcoma may arise de novo, may be synchronous multicentric (6), metastatic (7), or appear in benign precursor lesions, which may include cemento-osseous dysplasia (8) or ossifying fibroma (9). Cemento-osseous lesions are lesions of the jaw closely associated with the apices of the teeth that contain amorphous spherical calcifications resembling an aberrant form of cementum.

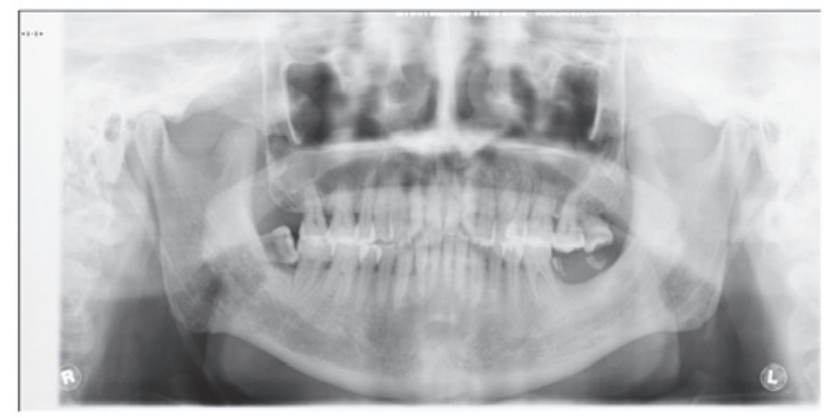

Figure 1. Panoramic X-ray revealed a radiopaque lesion adjacent to the roots of the teeth from the left maxillary incisor to the second bicuspid. The border of the mass was diffuse and irregular. Moreover, the porosity in the surrounding bone indicated an invasive process.
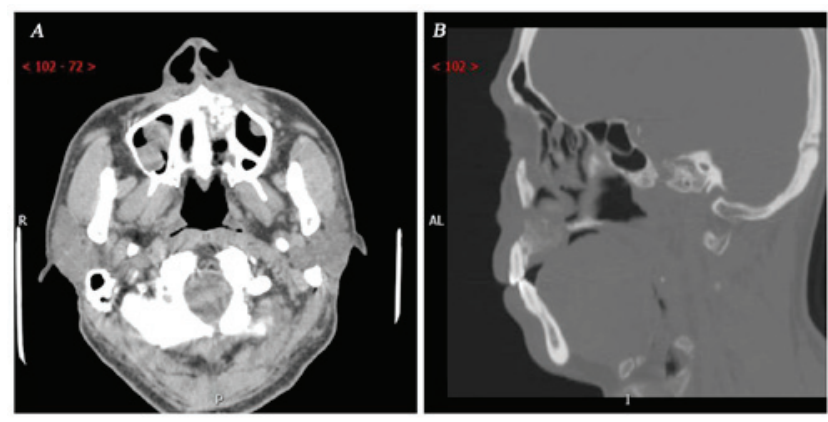

Figure 2. Computed tomography (CT) images. (A) Axial CT scan, soft tissue algorithm, showing an osteoblastic destructive mass extending in the nasal cavity and maxillary sinus, without periosteal reaction or soft tissue extension. (B) Multiplanar reformatted sagittal CT scan, bone algorithm, showing a destructive mass extending from the left alveolar ridge into the nasal cavity, with osteoid calcification.

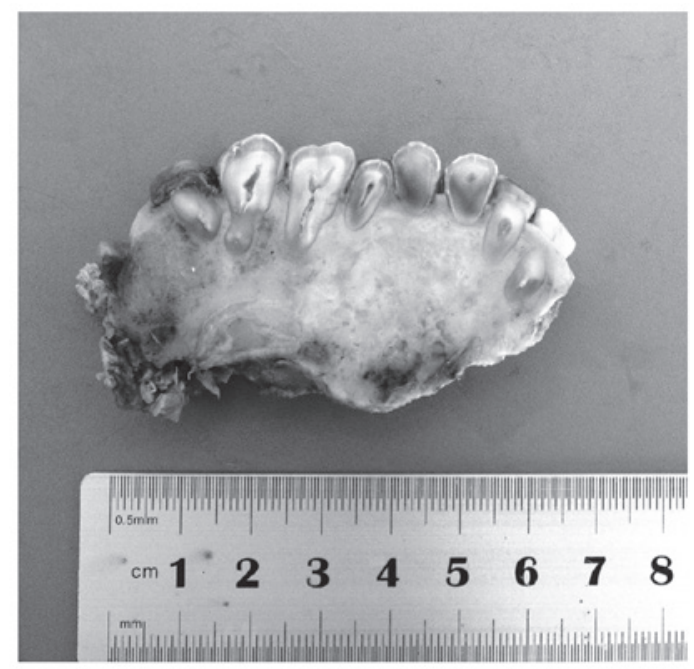

Figure 3. Macroscopic view of the surgical specimen of the left maxillary bone from the palatal view. The mass was pale, diffuse and irregular, and it involved a larger part of the bone than what appeared on radiographic images, including the alveolar ridge and the body of the maxilla from the first incisor to the first molar. On sectioning, the teeth roots were partially embedded in the tumor.

Specifically for this case, the differential diagnosis among primary osteosarcoma, concurrent cemento-osseous dysplasia and secondary malignant tumor in a background of 

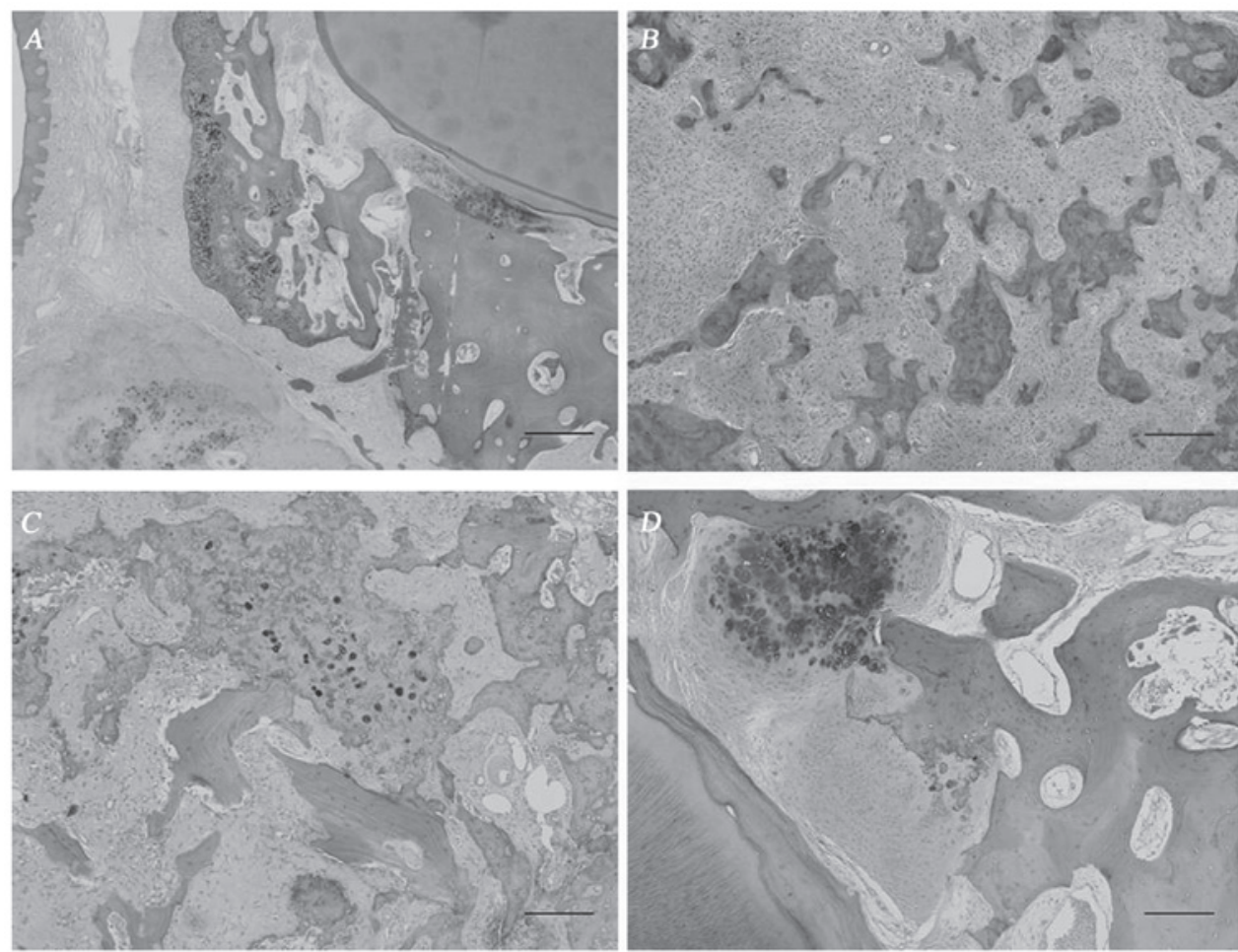

Figure 4. The morphology of the mass in the alveolar part of the left maxillary bone resembled a cemento-osseous lesion. (A) Interface of the tooth apex and the tumor. Low-power view of the tumor involving alveolar bone and periodontal ligament. (B) Abundant cementicle-like structures were formed among highly atypical spindle cells. Matrix was present between the tumor cells. (C) The tumor cells permeated between the preexisting trabeculae of the medullary bone. (D) The tumor was located on the side of alveolar bone, rather than the side of the cementum, which indicated its osteogenic nature. Hematoxylin and eosin staining; magnification, $x 10$.
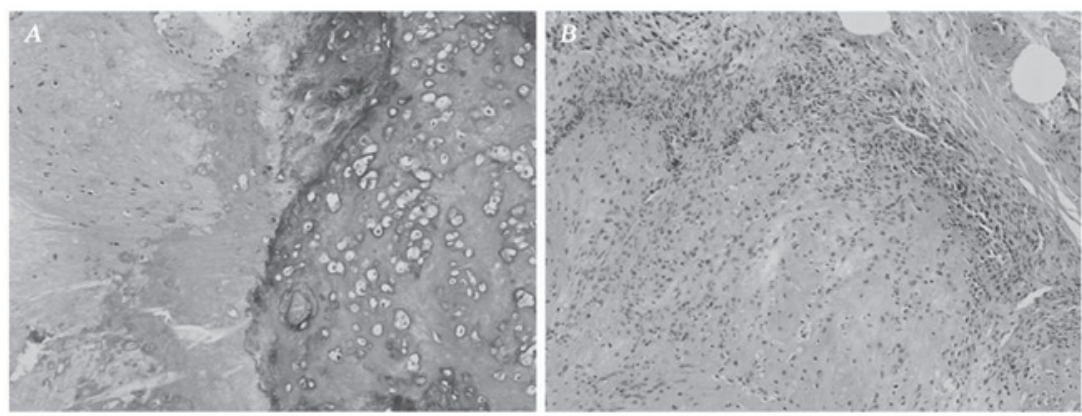

Figure 5. The histological appearance of the tumor in body of the left maxillary bone suggested a typical chondroblastic osteosarcoma. (A) The tumor contained deposits of hypercellular cartilage and abnormal osteoid and bone. (B) There was crowding at the periphery of the lobule, where sheets of atypical, pleomorphic and hyperchromatic sarcomatous cells were formed. Hematoxylin and eosin staining; magnification, x10.

cemento-osseous dysplasia or ossifying fibroma, may be difficult radiographically as well as histopathologically. Generally, the suspected clinical diagnosis was a cementum-originating tumor due to its association with the roots of the teeth and absence of periosteal reaction. The pathological appearance in the alveolar bone was confusing due to the presence of a cementicle-like structure. However, the typical manifestations in the body of the maxilla provided strong evidence for the diagnosis of primary chondroblastic osteosarcoma.

The mechanisms underlying the formation of bone, cartilage, or cementum in the oral area are helpful for understanding the nature of the lesions involving hard tissue formation, and may provide clues for differential diagnosis. Cells forming bone in the jaw may be divided into three types:
Osteoblasts lying on the bone surface, osteogenic fibroblasts in the periodontal ligament and periosteum, and non-specific fibroblasts stimulated to differentiate into osteogenic cells after degeneration (10). The first two cell types are associated with the origin of jaw osteosarcoma, whereas the osteogenic cells in the periodontal ligament produce cementum (11). Therefore, the tumor cells responsible for hard tissue formation in osteosarcoma, cemento-osseous dysplasia or ossifying fibroma, may share a common origin. This may explain the presence of the cementicle-like structure in this osteosarcoma. However, cementum is a bone-like tissue that covers the root of the tooth. Cementocytes are similar to osteocytes, but their cell processes tend to be orientated in one direction, namely towards the periodontal ligament, rather than equally around 
the cell body, as in osteocytes. Therefore, what was referred to as a cementicle-like structure in this case was in fact abnormal osteoid structure of osteosarcoma. Osteoid displays a woven or mat-like appearance, unlike the more orderly longitudinal fiber array found in collagen.

\section{References}

1. Sato K and Unni KK: Malignant tumors of bone and cartilage. In: Pathology and Genetics of Head and Neck Tumors: World Health Organization Classification of Tumors. Barnes L, Eveson JW, Reichart P, Sidransky D (eds). IARC Press, Lyon, pp7-52, 2005.

2. Nthumba PM: Osteosarcoma of the jaws: A review of literature and a case report on synchronous multicentric osteosarcomas. World J Surg Oncol 10: 240, 2012.

3. Forteza G, Colmenero B and López-Barea F: Osteogenic sarcoma of maxilla and mandible. Oral Surg Oral Med Oral Pathol 62: 179-184, 1986.

4. Paparella ML, Olvi LG, Brandizzi D, Keszler A, Santini-Araujo E and Cabrini RL: Osteosarcoma of the jaw: An analysis of a series of 74 cases. Histopathology 63: 551-557, 2013.

5. Wang S, Shi H and Yu Q: Osteosarcoma of the jaws: Demographic and CT imaging features. Dentomaxillofac Radiol 41: 37-42, 2012.

6. Jia S and Li B: Osteosarcoma of the Jaws: Case report on synchronous multicentric osteosarcomas. J Clin Diagn Res 8 : ZD01-ZD03, 2014.
7. Carnelio S, Pai K, Rao N, Solomon M and Ahasan A: Metastatic osteosarcoma to the maxilla: A case report and a review of the literature. Quintessence Int 33: 397-399, 2002.

8. Olusanya AA, Adeyemi BF and Adisa AO: Concurrent cemento-osseous dysplasia and osteogenic sarcoma: Report of two cases. Case Rep Med 2012: 180561, 2012.

9. Koury ME, Regezi JA, Perrott DH and Kaban LB: 'Atypical' fibro-osseous lesions: Diagnostic challenges and treatment concepts. Int J Oral Maxillofac Surg 24: 162-169, 1995.

10. Matsuzaka K, Shimono M, Uchiyama T, Noma H and Inoue T: Lesions related to the formation of bone, cartilage or cementum arising in the oral area: A statistical study and review of the literature. Bull Tokyo Dent Coll 43: 173-180, 2002.

11. Roguljic H, Matthews BG, Yang W, Cvija H, Mina M and Kalajzic I: In vivo identification of periodontal progenitor cells. J Dent Res 92: 709-715, 2013. 